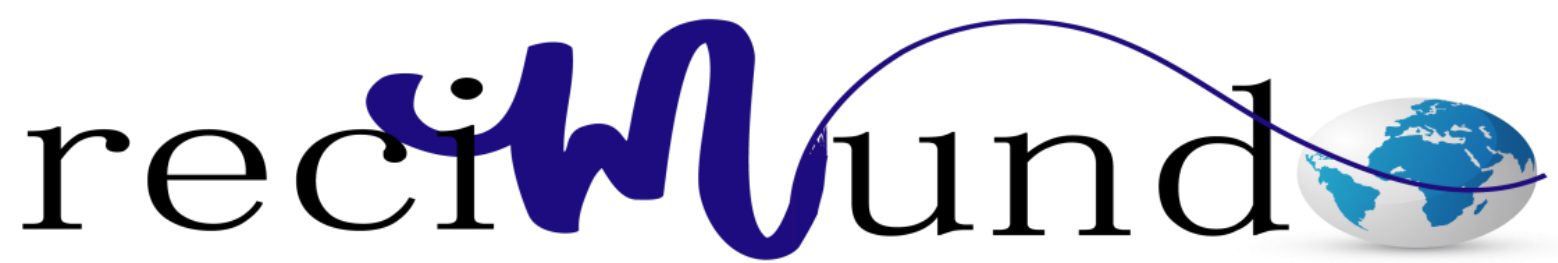

Revista Científica Mundo de la Investigación y el Conocimiento

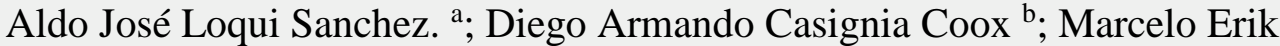

Zambrano Alarcon ${ }^{\text {c}}$; Freddy Gavilánez ${ }^{\text {d. }}$.

Indicadores bioproductivos y calidad de la canal en pollos camperos alimentados con maíz hidropónico con diferentes porcentajes de inclusión

Indicators bioproductive and carcass quality in pollos camperos hydroponic cornfed with different percentages of inclusion

Revista Científica Mundo de la Investigación y el Conocimiento. Vol. 3 núm.3, septiembre, ISSN: 2588-073X, 2019, pp. 699-716

DOI: $10.26820 /$ recimundo/3.(3).septiembre.2019.699-716

URL: $\underline{\text { http://recimundo.com/index.php/es/article/view/545 }}$

Código UNESCO: 3104 Producción Animal

Tipo de Investigación: Artículo de Revisión

Editorial Saberes del Conocimiento

Recibido: 15/05/2019

Aceptado: 23/06/2019

Publicado: 30/09/2019

Correspondencia: aldo loqui@hotmail.com

a. Magister en Riego y Drenaje; Ingeniero Agrónomo; Facultad de Medicina Veterinaria y Zootecnia, Universidad de Guayaquil; aldo_loqui@hotmail.com

b. Médico Veterinario y Zootecnista; Investigador Independiente; Guayaquil, Ecuador.

c. Magister en Educación Agropecuaria, mención Desarrollo Sostenible; Biólogo; Investigador Independiente; Guayaquil, Ecuador.

d. Investigador Independiente; Guayaquil, Ecuador. 


\section{Indicadores bioproductivos $y$ calidad de la canal en pollos camperos alimentados con maíz hidropónico con diferentes porcentajes de inclusión}

Vol. 3, núm. 3., (2019)

Aldo José Loqui Sanchez; Diego Armando Casignia Coox; Marcelo Erik Zambrano Alarcon; Freddy Gavilánez

\section{RESUMEN}

El presente trabajo tuvo como objetivo analizar las ventajas del uso de forraje de maíz hidropónica en pollos camperos tanto en la parte bioproductiva y organoléptica. En la presente investigación se evaluaron cuatro porcentajes de complementación de forraje de maíz hidropónico, con pollos de 28 días de edad durante 4 semanas, se utilizó un diseño al azar con sistema factorial de réplicas donde se trabajó con cinco tratamientos, y cuatro replicas, cada replica estuvo constituida por 10 aves, lo que llevaría a un total de 40 aves por tratamiento y 200 aves para el trabajo de investigación. Mostrando los siguientes resultados en la parte bioproductiva; ganancia media diaria y conversión acumulada. A 5\%: GMD (40,10), IC (2,4). B 8\%: GBM (41,02), IC (2,3). C 10\% GMD (39,61), IC (2,4). D 12\% GMD (40,21), IC (2,4). T GMD (41,43), IC (2,4).

En cuanto a los valores del rendimiento a la canal; Grupo A 5\% - 55,4\%. Grupo B 8\% -51,8\%. Grupo C 10\% - 44,7 \%. Grupo D 12\% - 50 \%. Grupo T Tratamiento- 58\%. Se presentó una mortalidad de un $6 \%$ enfocada solo en el grupo testigo. Los análisis no presentando diferencias significativas, a un máximo de $5 \%$ de probabilidad de error. En cuanto al análisis organolépticas, el análisis univariado presenta diferencias significativas ( $p>0,05)$ en las variables analizadas, a excepción del color en donde la probabilidad de error I es de 0,355.

Palabras clave: Pollo campero; Forraje de maíz hidropónico; Parámetros bioproductivos; Análisis organoléptico. 


\title{
Indicadores bioproductivos y calidad de la canal en pollos camperos alimentados con maíz hidropónico con diferentes porcentajes de inclusión
}

Vol. 3, núm. 3., (2019)

Aldo José Loqui Sanchez; Diego Armando Casignia Coox; Marcelo Erik Zambrano Alarcon;

Freddy Gavilánez

\begin{abstract}
This study aimed to analyze the advantages of the use of corn forage hydroponic in campers chickens both in the part bioproductiva and organoleptic. In the present investigation were evaluated four percentages of complementation of forage hydroponic corn, chickens of 28 days of age for 4 weeks, a random system design of mirrors where they worked with five treatments, and four factorials was used replicas, each replica was made up of 10 birds, which would lead to a total of 40 birds per treatment and 200 birds for research work. Showing the following results in the part bioproductiva; average daily gain and cumulative conversion. 5\%: GMD (40,10), IC (2.4). (B) 8\%: GBM (41,02), IC (2,3). C 10\% GMD (39,61), IC (2.4). D 12\% GMD (40,21), IC (2.4). T GMD (41.43), IC (2.4).

In terms of the values of the performance to the channel; Group to 5\% to 55.4\%. Group B 8-51.8\%. Group C 10\% - 44.7\%. Group D-12-50\%. Group T treatment-58\%. I present a mortality rate of $6 \%$ focused only in the control group. The analysis not showing significant differences, to a maximum of 5\% probability of error. Organoleptic analysis, univariate analysis shows significant differences $(p>0.05)$ in the variables analyzed, with the exception of the color where the probability of error I is of 0,355 .
\end{abstract}

Key words: Campers chickens; Forage hydroponic corn; Productive parameters; Organoleptic analysis. 


\section{Indicadores bioproductivos $y$ calidad de la canal en pollos camperos alimentados con maíz hidropónico con diferentes porcentajes de inclusión}

Vol. 3, núm. 3., (2019)

Aldo José Loqui Sanchez; Diego Armando Casignia Coox; Marcelo Erik Zambrano Alarcon; Freddy Gavilánez

\section{Introducción.}

A lo largos de los últimos años, la avicultura es el sector pecuario que más avances tecnológicos ha tenido a nivel mundial. La industria avícola es la que más futuro tiene a pesar de la desaceleración económica que se ha presentado en los últimos años. (Betancourt Rodríguez 2017)

El pollo Campero es un cruzamiento de razas productoras de carne con menor velocidad de crecimiento que las estirpes utilizadas en la producción de pollos parrilleros. Más allá de la menor velocidad de crecimiento que ostenta este tipo de ave respecto al pollo estándar comercial, su conformación posee relevancia debido a que su finalidad es la producción de carne y este es un carácter íntimamente relacionado con el programa de selección aplicado a los reproductores. (Sindik M et al. 2012)

El uso de forraje hidropónicos de maíz ha dado óptimos resultados tanto en animales monocavitatorios como poli-cavitatorios debido a que el animal consume todo el jergón formado por el forraje verde hidropónico, donde se encuentran las raíces, semillas sin germinar y la parte verde de la planta, este jergón aporta nutrientes como vitaminas, enzimas, coenzimas y aminoácidos libres. (Casignia C. 2018)

Las tendencias y exigencias actuales del mercado han llevado a aceptar tres conceptos de calidad: la calidad higiénico-sanitaria o seguridad del alimento, la calidad organoléptica o sensorial y la calidad nutricional, dictada por el valor nutritivo. De todos modos, hoy en día, la seguridad alimentaria y la palatabilidad son las propiedades en las cuales el consumidor pone más énfasis en 


\section{Indicadores bioproductivos y calidad de la canal en pollos camperos alimentados con maíz hidropónico con diferentes porcentajes de inclusión}

Vol. 3, núm. 3., (2019)

Aldo José Loqui Sanchez; Diego Armando Casignia Coox; Marcelo Erik Zambrano Alarcon;

Freddy Gavilánez

el momento de definir la preferencia en la compra de carne. Las propiedades organolépticas de la carne o de cualquier alimento en general, se definen como aquellos atributos percibidos por el consumidor en el momento de su consumo. Entre los atributos que más influyen en la satisfacción, destacan los relacionados con la textura o consistencia, caracterizados por las impresiones de terneza y jugosidad, y el sabor que reúne las sensaciones olfativas y gustativas, que es lo que denominamos gusto. (Xargayó et al. 2017)

\section{Materiales y métodos}

Locación. La investigación se realizó en la "Facultad de Medicina Veterinaria y Zootecnia" de la Universidad de Guayaquil en la provincia del Guayas, Republica del Ecuador, a una altura de 5 metros sobre el nivel del mar, con una temperatura promedio anual de $25.90{ }^{\circ} \mathrm{C}$, humedad relativa del $80 \%$, precipitación promedio anual de $1445 \mathrm{~m}$. m.

Diseño estadístico. Para la presente investigación se utilizó un diseño al azar con sistema factorial de réplicas donde se trabajó con cinco tratamientos, y cuatro replicas, cada replica estuvo constituida por 10 aves, lo que llevaría a un total de 40 aves por tratamiento y 200 aves para el trabajo de investigación en un tiempo total de 12 semanas.

Para realizar el análisis estadístico de la presente investigación se utilizaron dos softwares, el INFOSTAT (Di Rienzo 2009) para la parte estadística cuantitativa (conversión alimenticia), y IBM SPSS para la estadística cualitativa (sensorial organoléptica) con una estadística multivariada (MANOVA) con cuatro métodos estadísticos: Traza de Pillai, Lambda de Wilks, Traza de Hotelling y Raíz mayor de Roy.(Hurtado et al. 2004) Debido a que el trabo fue experimental se 


\section{Indicadores bioproductivos $y$ calidad de la canal en pollos camperos alimentados con maíz hidropónico con diferentes porcentajes de inclusión}

Vol. 3, núm. 3., (2019)

Aldo José Loqui Sanchez; Diego Armando Casignia Coox; Marcelo Erik Zambrano Alarcon; Freddy Gavilánez

utilizó una estadística no probabilística, por ello el diseño fue de bloques distribuidos completamente al azar con sistema factorial.

Para el estudio se utilizaron 5 grupos distribuidos en 4 tratamientos y un grupo control, representados de la siguiente manera:

- Grupo 1(A): alimentación más complementación del 5\% de FMH.

- Grupo 2 (B): alimentación más complementación 8\% de FMH

- Grupo 3(C): balanceado más complementación del 10\% de FMH

- Grupo 4(D): alimentación más complementación del 12\% de FMH

- Grupo 5 Testigo (T): alimentación sin complementación de FMH

Se realizaron cuatro repeticiones por tratamiento y grupo control.

Los cubículos fueron diseñados pensando en el espacio vital para los pollos el cual era de 8 aves X m2. Para el estudio se necesitó un total de 20 cubículos los cuales constaban las siguientes dimensiones: $1.20 \mathrm{~m}$ de largo X $0.8 \mathrm{~m}$ de ancho, con una altura de $1 \mathrm{~m}$ y una cama de $20 \mathrm{~cm}$ de espesor. Cada cubículo constaba con un bebedero automático y un comedero de campana.

Para la investigación se recibieron pollos de apenas 12 horas de nacidos, se realizó el manejo pertinente de estos lo cual conta de pesado y aplicación de vacunas, cabe destacar que las instalaciones ya estaban acondicionadas con anterioridad para el ingreso de las aves y se realizó su manejo de acuerdo a los manuales de aviares. (Aviagen 2014). Se realizó peso a los pollos por semana para medir su desarrollo, hasta que alcanzaron los 28 días de edad, hasta se momento los 


\section{Indicadores bioproductivos y calidad de la canal en pollos camperos alimentados con maíz hidropónico con diferentes porcentajes de inclusión}

Vol. 3, núm. 3., (2019)

Aldo José Loqui Sanchez; Diego Armando Casignia Coox; Marcelo Erik Zambrano Alarcon;

Freddy Gavilánez

pollos se mantenían en una sola área común, luego fueron separadas en sus distintos cubículos elegidas aleatoriamente 10 aves por cubículo, a los cuales se les asigno un nombre de registro para los tratamientos: Grupo A, Grupo B, Grupo C, Grupo D, Grupo T testigo o control, los cuales se describen a continuación:

Grupo A: A este grupo se le proporcionó alimento balanceado comercial de engorda más complementación de forraje hidropónico de maíz, el cual fue cortado en partículas pequeñas de no más de $0,5 \mathrm{~cm}$ de diámetro, este complemento se administró al 5\%.

Grupo B: A este grupo se le proporcionó alimento balanceado comercial de engorde más complementación de forraje hidropónico de maíz, el cual fue cortado en partículas pequeñas de no más de $0,5 \mathrm{~cm}$ de diámetro, este complemento se administró al $8 \%$.

Grupo C: A este grupo se le proporcionó alimento balanceado comercial de engorde más complementación de forraje hidropónico de maíz, el cual fue cortado en partículas pequeñas de no más de $0,5 \mathrm{~cm}$ de diámetro, este complemento se administró al $10 \%$.

Grupo D: A este grupo se le proporcionó alimento balanceado comercial de engorde más complementación de forraje hidropónico de maíz, el cual fue cortado en partículas pequeñas de no más de $0,5 \mathrm{~cm}$ de diámetro, este complemento se administró al $12 \%$.

Grupo T testigo o control: A este grupo se le proporciono alimento balanceado comercial de engorde sin la complementación de forraje hidropónico de maíz. 


\section{Indicadores bioproductivos $y$ calidad de la canal en pollos camperos alimentados con maíz hidropónico con diferentes porcentajes de inclusión}

Vol. 3, núm. 3., (2019)

Aldo José Loqui Sanchez; Diego Armando Casignia Coox; Marcelo Erik Zambrano Alarcon; Freddy Gavilánez

Para el complemento alimenticio se germino forraje de maíz hidropónico durante 14 días, el hibrido utilizado para la investigación fue el Trueno NB7443.

Medición y muestreo. Para el análisis de los parámetros bioproductivos se estudió el rendimiento a la canal, la mortalidad, la conversión alimenticia y ganancia media diaria (GMD). En cuanto al estudio organoléptico de la carne se procedieron a medir las variables: apariencia de la carne, color, olor, sabor, consistencia, textura, jugosidad. Los datos fueron analizados por 10 catadores usando los siguientes rangos.

Tabla 1. valoración de la carne para las distintas variables dadas por los catadores.

\begin{tabular}{|ll|}
\hline - & Me disgusta mucho. (1) \\
\hline - & No me gusta. (2) \\
\hline - & Me resulta indiferente. (3) \\
\hline - & Me gusta. (4) \\
\hline - & Me gusta mucho. (5) \\
\hline
\end{tabular}

\section{Resultados}

A continuación, se presentan los resultados obtenidos de las variables de estudios, al alimentar a los pollos camperos con una dieta de 5, 8, 10 y 12\% con FMH como complemento a la dieta de engorde.

La mortalidad registrada fue del $6 \%$ enfocada únicamente al grupo testigo. 


\section{Indicadores bioproductivos y calidad de la canal en pollos camperos alimentados con maíz hidropónico con diferentes porcentajes de inclusión}

Vol. 3, núm. 3., (2019)

Aldo José Loqui Sanchez; Diego Armando Casignia Coox; Marcelo Erik Zambrano Alarcon;

Freddy Gavilánez

Tabla 1. Ganancia media diaria e índice de conversión acumulada

\begin{tabular}{|c|c|c|}
\hline Grupo & GMD/gr & IC \\
\hline T & 41,43 & 2,4 \\
\hline A 5\% & 40,10 & 2,4 \\
\hline B 8\% & 41,02 & 2,3 \\
\hline C 10\% & 39,61 & 2,4 \\
\hline D 12\% & 40,21 & 2,4 \\
\hline
\end{tabular}

Tabla 2. Rendimiento a la canal

\begin{tabular}{|c|c|}
\hline \% de inclusión & Rendimiento a la canal \\
\hline Grupo A 5\% & $55,4 \%$ \\
\hline Grupo B 8\% & $51,8 \%$ \\
\hline Grupo C 10\% & $44,7 \%$ \\
\hline Grupo D 12\% & $50 \%$ \\
\hline Grupo T Tratamiento & $58 \%$ \\
\hline
\end{tabular}

Tabla 3. Resultados estadísticos del peso de las aves.

\begin{tabular}{|c|c|c|c|c|c|c|c|}
\hline \multicolumn{8}{|c|}{ Peso de los pollos (g) } \\
\hline \multirow{2}{*}{$\mathrm{N}^{\circ}$} & \multirow{2}{*}{$\begin{array}{l}\text { Concentración de } \\
\text { FHM }(\%) \text { (Factor } \\
\text { A) }\end{array}$} & \multirow{2}{*}{$\begin{array}{l}\text { Periodos } \\
\text { (Factor B) }\end{array}$} & \multicolumn{4}{|c|}{ Repeticiones } & \multirow{2}{*}{ Promedios } \\
\hline & & & I & II & III & IV & \\
\hline 1 & Grupo A 5\% & 11/octubre & 1015,0 & 947,5 & 945,0 & 982,5 & 972,5 \\
\hline 2 & Grupo A 5\% & 19/octubre & 1330,0 & 1265,0 & 1280,0 & 1290,0 & 1291,3 \\
\hline 3 & Grupo A 5\% & 27/octubre & 1785,0 & 1685,0 & 1655,0 & 1735,0 & 1715,0 \\
\hline 4 & Grupo A 5\% & 06/noviembre & 2416,7 & 2200,0 & 2265,0 & 2320,0 & 2300,4 \\
\hline 5 & Grupo B 8\% & 11/octubre & 1020,0 & 942,5 & 992,5 & 985,0 & 985,0 \\
\hline 6 & Grupo B 8\% & 19/octubre & 1320,0 & 1255,0 & 1303,0 & 1320,0 & 1299,5 \\
\hline 7 & Grupo B 8\% & 27/octubre & 1745,0 & 1695,0 & 1735,0 & 1745,0 & 1730,0 \\
\hline 8 & Grupo B 8\% & 06/noviembre & 2335,0 & 2280,0 & 2330,0 & 2455,0 & 2350,0 \\
\hline 9 & Grupo C $10 \%$ & 11/octubre & 880,0 & 947,5 & 970,0 & 922,5 & 930,0 \\
\hline 10 & Grupo C $10 \%$ & 19/octubre & 1175,0 & 1295,0 & 1300,0 & 1255,0 & 1256,3 \\
\hline 11 & Grupo C 10\% & 27/octubre & 1560,0 & 1695,0 & 1715,0 & 1665,0 & 1658,8 \\
\hline 12 & Grupo C $10 \%$ & 06/noviembre & 2110,0 & 2295,0 & 2360,0 & 2330,0 & 2273,8 \\
\hline
\end{tabular}


Indicadores bioproductivos y calidad de la canal en pollos camperos alimentados con maíz hidropónico con diferentes porcentajes de inclusión

Vol. 3, núm. 3., (2019)

Aldo José Loqui Sanchez; Diego Armando Casignia Coox; Marcelo Erik Zambrano Alarcon; Freddy Gavilánez

\begin{tabular}{|l|l|l|l|l|l|l|l|}
\hline 13 & Grupo D 12\% & $11 /$ octubre & 935,0 & 965,0 & 972,5 & 880,0 & 938,1 \\
\hline 14 & Grupo D 12\% & $19 /$ octubre & 1260,0 & 1265,0 & 1295,0 & 1280,0 & 1275,0 \\
\hline 15 & Grupo D 12\% & $27 /$ octubre & 1695,0 & 1715,0 & 1710,0 & 1670,0 & 1697,5 \\
\hline 16 & Grupo D 12\% & 06/noviembre & 2365,0 & 2283,3 & 2333,3 & 2244,4 & 2306,5 \\
\hline 17 & Grupo testigo & $11 /$ octubre & 980,0 & 970,0 & 970,0 & 990,0 & 977,5 \\
\hline 18 & Grupo testigo & $19 /$ octubre & 1300,0 & 1300,0 & 1285,0 & 1305,0 & 1297,5 \\
\hline 19 & Grupo testigo & 27/octubre & 1750,0 & 1777,8 & 1410,0 & 1783,3 & 1680,3 \\
\hline 20 & Grupo testigo & 06/noviembre & 2478,6 & 2366,7 & 2366,7 & 2277,8 & 2372,4 \\
\hline
\end{tabular}

Datos promedios realizados en los diferentes tratamientos por fechas

Análisis estadístico

Análisis de la varianza

Variable N $\mathrm{R}^{2} \mathrm{R}^{2} \mathrm{Aj} \mathrm{CV}$

Peso (g) $800,99 \quad 0,984,33$

Cuadro de Análisis de la Varianza (SC tipo III)

\begin{tabular}{lrrrrr}
\multicolumn{1}{c}{ F.V. } & \multicolumn{1}{c}{ SC } & gl & \multicolumn{1}{c}{ CM } & \multicolumn{1}{c}{ F } & p-valor \\
\hline Modelo. & 20706990,90 & 22 & 941226,86 & $204,62<0,0001$ \\
Concentración de maíz & 37653,85 & 4 & 9413,46 & 2,05 & 0,1000 \\
Fechas & 20649919,30 & 3 & 6883306,43 & 1496,45 & $<0,0001$ \\
Concentración de maíz*Fech. & 15535,36 & 12 & 1294,61 & 0,28 & 0,9901 \\
Repeticiones & 3882,39 & 3 & 1294,13 & 0,28 & 0,8386 \\
Error & 262186,61 & 57 & 4599,77 & & \\
& & & \\
Total & 20969177,50 & 79 \\
\hline
\end{tabular}

Test de comparación de Duncan para las concentraciones de FHM (maíz)

Test:Duncan Alfa=0,05

Error: 4599,7650 gl: 57

Concentración de maíz Medias n E.E.

a2: grupo B 8\% 1591,13 16 16,96 A

a5: grupo testigo $1581,931616,96 \mathrm{~A}$

a1: grupo A $5 \% \quad 1569,791616,96$ A B

a4: grupo D $12 \% \quad 1554,281616,96$ A B

a3: grupo C 10\% $1529,691616,96 \quad \mathrm{~B}$

Medias con una letra común no son significativamente diferentes $(p>0,05)$

Test de comparación de Duncan para las fechas de evaluación.

Test:Duncan Alfa=0,05

Error: $4599,7650 \mathrm{gl}: 57$

Fechas Medias n E.E.

b4: 06/noviembre 2320,6320 15,17 A

b3: 27/octubre 1696,312015,17 B

b2: 19/octubre 1283,902015,17 C

b1: 11/octubre $960,632015,17 \quad$ D

Medias con una letra común no son significativamente diferentes ( $p>0,05$ )

Revista Científica Mundo de la Investigación y el Conocimiento. 3 (3). pp. 699-716 


\section{Indicadores bioproductivos y calidad de la canal en pollos camperos alimentados con maíz hidropónico con diferentes porcentajes de inclusión}

Vol. 3, núm. 3., (2019)

Aldo José Loqui Sanchez; Diego Armando Casignia Coox; Marcelo Erik Zambrano Alarcon;

Freddy Gavilánez

Los tratamientos (concentraciones de FHM) no presentan diferencias significativas, a un máximo de 5\% de probabilidad de error; sin embargo, al existir similitudes entre los promedios con respecto al tratamiento testigo, esto permite colegir que la alimentación puede ser sustituida hasta en un $12 \%$ FHM.

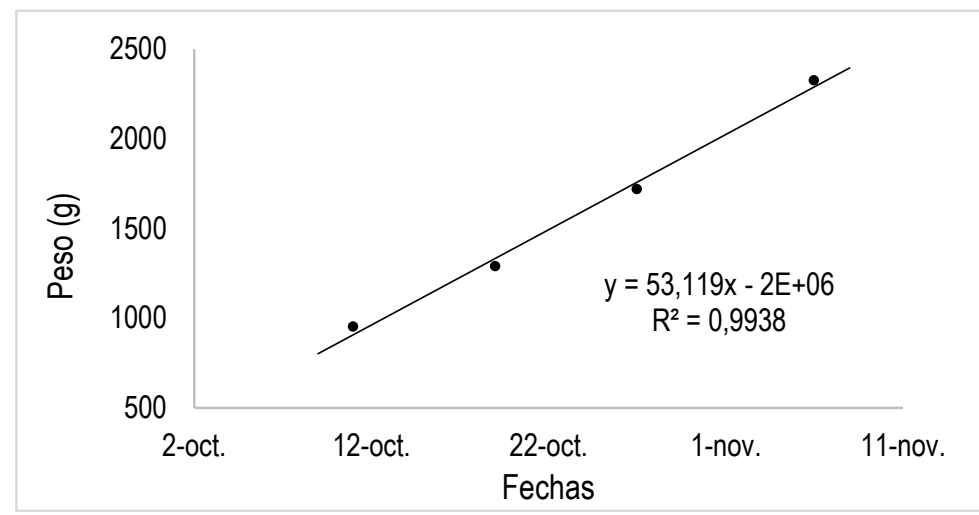

Durante el tiempo del estudio, el crecimiento obtenido en los pollos se ajusta a un modelo de regresión lineal, en la cual el incremento de peso diario fue de 53,1 g.

Análisis estadístico de las características sensoriales.

Dada la relación existente en el criterio de las variables Apariencia, Olor, Color, Consistencia, Sabor, Aroma, Textura y Jugosidad, la valoración estadística correspondiente realizada es un Análisis de varianza multivariado (MANOVA) considerando estas ocho variables. Este resultado es que se detalla a continuación: 
Indicadores bioproductivos y calidad de la canal en pollos camperos alimentados con maíz hidropónico con diferentes porcentajes de inclusión

Vol. 3, núm. 3., (2019)

Aldo José Loqui Sanchez; Diego Armando Casignia Coox; Marcelo Erik Zambrano Alarcon; Freddy Gavilánez

Tabla 5. Análisis de varianza multivariado para las variables Apariencia, Olor, Color,

Consistencia, Sabor, Aroma, Textura y Jugosidad.

\begin{tabular}{|c|c|c|c|c|c|c|}
\hline \multicolumn{7}{|c|}{$\begin{array}{c}\text { Contarstes } \\
\text { multivariados }\end{array}$} \\
\hline \multicolumn{2}{|l|}{ Efecto } & valor & $\mathrm{F}$ & $\begin{array}{c}\text { Gl de la } \\
\text { hipótesis }\end{array}$ & Gl del error & Sig. \\
\hline \multirow[b]{4}{*}{ Intersección } & Traza de Pillai & ,997 & $1102,334 b$ & 8,000 & 29,000 &, 000 \\
\hline & Lambda de Wilks & ,003 & $1102,334 b$ & 8,000 & 29,000 & ,000 \\
\hline & Traza de Hotelling & 304,092 & $1102,334 b$ & 8,000 & 29,000 & ,000 \\
\hline & Raíz mayor de Roy & 304,092 & $1102,334 b$ & 8,000 & 29,000 & ,000 \\
\hline \multirow[b]{4}{*}{ Tratamientos } & Traza de Pillai & 1,294 & 1,913 & 32,000 & 128,000 & ,006 \\
\hline & Lambda de Wilks & , 108 & 2,811 & 32,000 & 108,542 & ,000 \\
\hline & Traza de Hotelling & 5,033 & 4,325 & 32,000 & 110,000 & ,000 \\
\hline & Raíz mayor de Roy & 4,433 & $17,733 \mathrm{c}$ & 8,000 & 32,000 &, 000 \\
\hline \multirow[b]{4}{*}{ Repeticiones } & Traza de Pillai & 2,055 & 1,383 & 288,000 & 288,000 & ,034 \\
\hline & Lambda de Wilks &, 065 & 1,454 & 183,000 & 183,000 &, 024 \\
\hline & Traza de Hotelling & 3,924 & 1,485 & 218,000 & 218,000 & 016 \\
\hline & Raíz mayor de Roy & 1,783 & $7,130 \mathrm{c}$ & 9,000 & 36,000 &, 000 \\
\hline \multicolumn{7}{|c|}{$\begin{array}{l}\text { a. Diseño: Intersección + Tratamientos + } \\
\text { Repeticiones } \\
\text { b. Estadistico exacto }\end{array}$} \\
\hline
\end{tabular}

Según el análisis multivariado indicado en la tabla 5, las variables están relacionadas y todas producen efectos significativos, de acuerdo a los cuatro estadígrafos que utiliza el software (Traza de Pillai, Lambda de Wilks, Traza de Hotelling y Raíz mayor de Roy). En cuanto al análisis univariado detallado en la tabla 6 , los tratamientos presentan diferencias significativas $(\mathrm{p}>0,05)$ en las variables analizadas, a excepción del color en donde la probabilidad de error I es de 0,355. Consecuentemente, en cada uno de las comparaciones realizadas con el test de Tukey al 5\% de probabilidad, el tratamiento que se diferencia del resto en cada una de las variables es aquel identificado como Grupo C (10\%), presentando promedios entre 4 y 5 de la escala utilizada. 
Indicadores bioproductivos y calidad de la canal en pollos camperos alimentados con maíz hidropónico con diferentes porcentajes de inclusión

Vol. 3, núm. 3., (2019)

Aldo José Loqui Sanchez; Diego Armando Casignia Coox; Marcelo Erik Zambrano Alarcon;

Freddy Gavilánez

Tabla 6. Análisis de varianza univariado para las variables Apariencia, Olor, Color,

Consistencia, Sabor, Aroma, Textura y Jugosidad.

\begin{tabular}{|c|c|c|c|c|c|c|}
\hline \multicolumn{7}{|c|}{$\begin{array}{c}\text { Prueba de los } \\
\text { efectos inter-sujetos }\end{array}$} \\
\hline \multirow{9}{*}{$\begin{array}{l}\text { origen } \\
\text { Modelo } \\
\text { corregido }\end{array}$} & $\begin{array}{l}\text { Variable } \\
\text { dependiente }\end{array}$ & $\begin{array}{c}\text { Suma de } \\
\text { cuadrados } \\
\text { tipo III }\end{array}$ & $\mathrm{gl}$ & $\begin{array}{c}\text { Medias } \\
\text { cuadráticas }\end{array}$ & $\mathrm{F}$ & Sig. \\
\hline & Apariencia & $7,000 a$ & 13 & ,538 & 2,154 & ,035 \\
\hline & Olor & $25,740 b$ & 13 & 1,980 & 7,850 & ,000 \\
\hline & Color & $5,160 c$ & 13 & ,397 & 1,219 & ,306 \\
\hline & Consistencia & $9,740 d$ & 13 & ,749 & 4,597 & ,000 \\
\hline & Sabor & $17,640 \mathrm{e}$ & 13 & 1,357 & 5,280 & ,000 \\
\hline & Aroma & $15,000 f$ & 13 & 1,154 & 5,934 & ,000 \\
\hline & Textura & $16,200 \mathrm{~g}$ & 13 & 1,246 & 5,751 & ,000 \\
\hline & jugosidad & $21,340 \mathrm{~h}$ & 13 & 1,642 & 4,661 & ,000 \\
\hline \multirow[t]{8}{*}{ Intersección } & Apariencia & 648,000 & 1 & 648,000 & 2592,000 & ,000 \\
\hline & Olor & 824,180 & 1 & 824,180 & 3267,674 & ,000 \\
\hline & Color & 677,120 & 1 & 677,120 & 2079,891 & ,000 \\
\hline & Consistencia & 699,380 & 1 & 699,380 & 4281,918 & ,000 \\
\hline & Sabor & 865,280 & 1 & 865,280 & 3430,626 & ,000 \\
\hline & Aroma & 648,000 & 1 & 648,000 & 3332,571 & ,000 \\
\hline & Textura & 882,000 & 1 & 882,000 & 4070,769 & ,000 \\
\hline & jugosidad & 744,980 & 1 & 744,980 & 2115,085 & ,000 \\
\hline \multirow[t]{8}{*}{ Tratamiento } & Apariencia & 4,600 & 4 & 1,150 & 4,600 & ,004 \\
\hline & Olor & 14,920 & 4 & 3,730 & 14,789 & ,000 \\
\hline & Color & 1,480 & 4 & ,370 & 1,137 & ,355 \\
\hline & Consistencia & 6,520 & 4 & 1,630 & 9,980 & ,000 \\
\hline & Sabor & 12,920 & 4 & 3,230 & 12,806 & ,000 \\
\hline & Aroma & 11,800 & 4 & 2,950 & 15,171 & ,000 \\
\hline & Textura & 9,800 & 4 & 2,450 & 11,308 & ,000 \\
\hline & jugosidad & 15,320 & 4 & 3,830 & 10,874 & ,000 \\
\hline
\end{tabular}

Revista Científica Mundo de la Investigación y el Conocimiento. 3 (3). pp. 699-716 


\section{Indicadores bioproductivos $y$ calidad de la canal en pollos camperos alimentados con maíz hidropónico con diferentes porcentajes de inclusión}

Vol. 3, núm. 3., (2019)

Aldo José Loqui Sanchez; Diego Armando Casignia Coox; Marcelo Erik Zambrano Alarcon; Freddy Gavilánez

Tabla 7. Análisis de varianza para la variable Masticabilidad.

Masticabilidad

DHS de Tukey' ${ }^{a, b}$
\begin{tabular}{|l|r|r|r|r|}
\hline & & \multicolumn{3}{|c|}{ Subconjunto } \\
\cline { 3 - 5 } Tratamientos & N & 1 & \multicolumn{1}{c|}{2} & \multicolumn{1}{c|}{3} \\
\hline Grupo D (12\%) & 10 & 1,3000 & & \\
Grupo C (10\%) & 10 & 1,8000 & & \\
Grupo B (8\%) & 10 & & 2,5000 & \\
Grupo A (5\%) & 10 & & 2,7000 & 2,7000 \\
Grupo testigo & 10 & & & 3,2000 \\
Sig. & &, 259 &, 921 &, 259 \\
\hline
\end{tabular}

Pruebas de los efectos inter-sujetos

Variable dependiente: Masticabilidad
\begin{tabular}{|l|r|r|r|r|r|}
\hline & $\begin{array}{c}\text { Suma de } \\
\text { cuadrados } \\
\text { tipo III }\end{array}$ & gl & $\begin{array}{c}\text { Media } \\
\text { cuadrática }\end{array}$ & \multicolumn{1}{c|}{ F } & \multicolumn{1}{c|}{ Sig. } \\
\hline Origen & $31,900^{\text {a }}$ & 13 & 2,454 & 8,334 &, 000 \\
Modelo corregido & 264,500 & 1 & 264,500 & 898,302 &, 000 \\
Intersección & 22,600 & 4 & 5,650 & 19,189 &, 000 \\
Tratamientos & 9,300 & 9 & 1,033 & 3,509 &, 003 \\
Repeticiones & 10,600 & 36 &, 294 & & \\
Error & 307,000 & 50 & & & \\
Total & 42,500 & 49 & & & \\
Total corregida & & & & \\
\hline
\end{tabular}

Según el análisis de varianza para la variable Masticabilidad, entre los tratamientos evaluados existen diferencias significativas (Tabla 7); y de acuerdo al test de Tukey, los tratamientos que presentaron las medias estadísticas menores fueron aquellos definidos como Grupo D (12\%) y Grupo C (10\%), presentando valores próximos a 1 y a 2 según la escala utilizada, respectivamente. 


\section{Indicadores bioproductivos y calidad de la canal en pollos camperos alimentados con maíz hidropónico con diferentes porcentajes de inclusión}

Vol. 3, núm. 3., (2019)

Aldo José Loqui Sanchez; Diego Armando Casignia Coox; Marcelo Erik Zambrano Alarcon;

Freddy Gavilánez

\section{Conclusión}

Los resultados obtenidos en las variables ganancia media diaria y rendimiento a la canal demostraron que los grupos a los cual se les aplico el complemento en sus distintos porcentajes no dieron a resaltar una variación significativa en sus pesos y una ligera variación en el rendimiento a la canal.

Con respecto a la mortalidad se puede verificar que se presentó mortalidad significativa en el grupo testigo, lo cual puede indicar que la alimentación con hidroponía de maíz puede influenciar el aumento inmunológico de las aves.

Los análisis organolépticos dieron como resultado que el uso de forrajes de maíz hidropónica arrojos buenos resultados con un 10\% y 12\% de complementación lo que dio mejoría a la mejoría al sabor de la carne de pollo.

\section{Discusión}

Al término de la investigación se obtuvieron datos estadísticos significativos en las variables estudiadas, se observó que los pesos obtenidos en el pollo campero complementados con ciertos porcentajes de forraje de maíz hidropónico tienden a presentar resultados altamente significativos $(\mathrm{p}<0,05)$ en el desarrollo de las aves demostrando que el alimentar el pollos camperos con forraje es viable y lo podemos constatar con la investigación (Andrade Yucailla et al. 2016) El cual presenta resultados significativos en el desarrollo de los pollos camperos. 


\section{Indicadores bioproductivos y calidad de la canal en pollos camperos alimentados con maíz hidropónico con diferentes porcentajes de inclusión}

Vol. 3, núm. 3., (2019)

Aldo José Loqui Sanchez; Diego Armando Casignia Coox; Marcelo Erik Zambrano Alarcon; Freddy Gavilánez

La variación de los porcentajes de inclusión tiende a provocar un notable cambio en los resultados obtenidos de las variables estudiadas, presentando valores significativos en el porcentaje del 8\% en ganancia media diaria (GMD) e índice de conversión acumulada (IC) los resultados de la investigación de (Leyva Cambar, Olmo González, and León Álvarez 2012) al usar forraje de morera en un 10, 20 y $30 \%$ demostraron que la aplicación de forrajes en las los pollos camperos tiende a dar buenos resultados en sus parámetros bioproductivos dándoles buenos resultados al $10 \%$ de aplicación.

Al alimentar con FMH se pudo verificar la capacidad que tiene este complemento alimentario en las propiedades organoléptica de la carne, el uso de porcentajes de 8 y $10 \%$ demuestran una evidente significancia según los análisis estudiados lo cual es un claro indicados de la efectividad del uso de FMH. Investigaciones como la de (Solorzano Saldarriaga 2016) en la que aplican infusiones de (mentha spicata) en distintas proporciones con el fin de obtener buenos resultados en los parámetros organolépticas. Por otro lado, la investigación de (Canet, Dottavio, and Di Masso 2015) en el cual compara dos genotipos de pollos camperos con alimento comercial, no mostrando resultados significativos a comparación de los efectos que ocasionan la aplicación de complementos o suplementos en la dieta animal. 


\section{Indicadores bioproductivos y calidad de la canal en pollos camperos alimentados con maíz hidropónico con diferentes porcentajes de inclusión}

Vol. 3, núm. 3., (2019)

Aldo José Loqui Sanchez; Diego Armando Casignia Coox; Marcelo Erik Zambrano Alarcon;

Freddy Gavilánez

\section{Referencias Bibliográficas}

Andrade Yucailla, V. et al. 2016. "Evaluación de dos fenotipos de pollos camperos en un sistema semi-intensivo con pastoreo en arachis pintoi en la amazonia ecuatoriana." Actas Iberoamericanas en Conservación Animal 8(January): 20-26. https://www.researchgate.net/profile/Raciel_Lima_Orozco/publication/316526614_Assessment_ of_two_free-range_chickens_phenotypes_in_a_semiintensive_system_with_Arachis_pintoi_grazing_in_ecuadorian_Amazonia/links/590229940f7e9 bcf654906b8/Assessment-of-two-f.

Aviagen. 2014. Manual de Manejo Del Pollo de Engorde Ross. http://es.aviagen.com/assets/Tech_Center/BB_Foreign_Language_Docs/Spanish_TechDocs/Ros sBroilerHandbook2014-ES.pdf.

Betancourt Rodríguez, Susana Andrea. 2017. "Efecto de Un Núcleo de Integridad Intestinal En Pollos de Engorde En La Avícola" Megaves" Ubicada En El Sector de Ascázubi, Provincia de Pichincha-Ecuador." http://dspace.udla.edu.ec/handle/33000/7503.

Canet, Zulma Edith, A M Dottavio, and R J Di Masso. 2015. "Análisis Sensorial Del Corte PataMuslo de Dos Genotipos de Pollo Campero Bajo Dos Manejos de La Alimentación." In Congreso Argentino de Producción Animal. 38. 201509 23-25, 23 Al 25 de Septiembre 2015. Santa Rosa, La Pampa. AR., http://ppct.caicyt.gov.ar/index.php/rapa/index.

Casignia C., Diego A. 2018. “'INDICADORES BIOPRODUCTIVOS Y CALIDAD DE LA CANAL EN POLLOS CAMPEROS ALIMENTADOS CON MAIIZ HIDROPÓNICO CON DIFERENTES PORCENTAJES DE INCLUSIÓN."' UNIVERSIDAD DE GUAYAQUIL. http://repositorio.ug.edu.ec/bitstream/redug/31227/1/CASIGNIA COOX DIEGO.pdf.

Hurtado, Ernesto et al. 2004. "Estudio Morfológico Del Cerdo Criollo Del Estado Apure, Venezuela." Revista computarizada de produccion porcina 11(3).

Leyva Cambar, Laercis, Carlos Olmo González, and Exequiel León Álvarez. 2012. "Inclusión de Harina Deshidratada de Follaje de Morera (Morus Alba L.) En La Alimentación Del Pollo Campero." En: Revista Científica UDO Agrícola 12(3): 653-59. http://ojs.udo.edu.ve/index.php/udoagricola/article/view/3028/24792593.

Di Rienzo, Julio A. 2009. "InfoStat Versión 2009. Grupo InfoStat, FCA, Universidad Nacional de Córdoba, Argentina." http://www. infostat. com. ar.

Sindik M et al. 2012. "Rendimiento a La Faena En Pollos Provenientes De Dos Genotipos De Reproductores Campero Inta Yield At Slaughter of Chickens From Crosses Involving Two Maternal Genotypes of Campero Inta Breeders.” Actas Iberoamericanas de Conservación Animal 
Indicadores bioproductivos y calidad de la canal en pollos camperos alimentados con maíz hidropónico con diferentes porcentajes de inclusión

Vol. 3, núm. 3., (2019)

Aldo José Loqui Sanchez; Diego Armando Casignia Coox; Marcelo Erik Zambrano Alarcon; Freddy Gavilánez

2: 279-81

http://www.uco.es/conbiand/aica/templatemo_110_lin_photo/articulos/2012/Trabajo039_AICA2 012.pdf.

Solorzano Saldarriaga, Julia Cristina. 2016. "Efectos de La Infusión de Mentha Spicata En Los Parámetros Productivos e Indicadores Organolépticos de La Canal En Pollos Broilers." http://repositorio.utmachala.edu.ec/bitstream/48000/7685/1/DE00042_TRABAJODETITULACI ON.pdf.

Xargayó, Marta et al. 2017. “Marinado Por Efecto 'Spray’: Una Solución Definitiva Para Mejorar La Textura de La Carne.” Recuperado el: 7-24. 